> Le génie tissulaire est un nouveau domaine, qui permet l'étude des mécanismes physiologiques du vivant. II s'agit d'une technologie fondée sur la capacité des cellules vivantes, en présence ou non de biomatériaux, à s'assembler en un tissu tridimensionnel. Elle constitue une voie intéressante ouvrant aux chercheurs la possibilité de considérer les cellules dans un contexte proche de celui retrouvé in vivo. Cet article résume les travaux en génie tissulaire menés par le laboratoire d'organogenèse expérimentale (LOEX) au cours des dernières années, dans le but de comprendre certains des mécanismes physiologiques et pathologiques de l'organisme humain. Ainsi, la cicatrisation cutanée, mais aussi les cellules souches, l'angiogenèse et les interactions cellulaires sont des secteurs ayant profité de l'apport du génie tissulaire. <

\section{Le génie tissulaire au service de la compréhension du vivant}

\author{
Véronique Moulin, Francine Goulet, \\ François Berthod, Lucie Germain, \\ François A. Auger
}

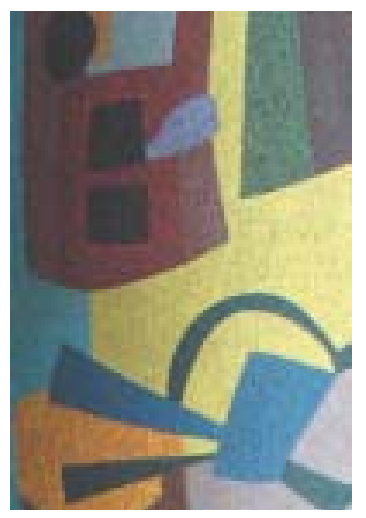

Laboratoire d'organogenèse expérimentale (LOEX), Hôpital du Saint-Sacrement, Centre hospitalier affilié universitaire de Québec, 1050, Chemin Sainte-Foy, Québec, Canada GIS 4L8, et Départements de chirurgie et de physiothérapie, Faculté de

Cependant, ces études médecine, Université Laval, ont été limitées par le nombre important de Québec, Canada. www.loex.ulaval.ca paramètres intervenant in vivo pour un mécanisme donné. Pour palier ces inconvénients, les études in vitro, c'està-dire utilisant des cellules humaines isolées et mises en culture, sont de plus en plus nombreuses. Ces études sont très intéressantes en ce qu'elles ont permis, là encore, d'étudier et d'élucider un certain nombre de mécanismes. Mais elles présentent le problème inverse des études réalisées sur le vivant: les modèles sont trop simples et ne reflètent pas, ou pas assez, les phénomènes observés in vivo. De plus, ces cellules sont très souvent cultivées en monocouche, ce qui ne reproduit pas l'environnement habituel des cellules dans l'organisme humain où elles se déploient généralement dans une structure tridimensionnelle.

Le génie tissulaire est une discipline en plein essor. Nouveau champ de connaissance, il tire parti de la capacité des cellules vivantes, qu'elles soient en présence ou non de biomatériaux, de s'assembler en un tissu tridimensionnel. Il ouvre une voie intéressante permettant aux chercheurs d'étudier les cellules dans un contexte proche de celui que l'on trouve in vivo. 
C'est donc un avantage par rapport à la culture classique de cellule in vitro. Les tissus ainsi créés sont cependant plus simples que ceux provenant des systèmes in vivo, car les conditions générales, ainsi que le nombre de types cellulaires et de paramètres, sont plus restreints et par là mieux maîtrisés.

\section{Les cellules jouent à saute-mouton}

Outre son rôle esthétique, la peau prévient la perte de fluides corporels et l'entrée de toxines et de microorganismes dans le corps, la perte de son intégrité ayant évidemment des conséquences néfastes. La réponse $d u$ corps à toute lésion est cruciale. Ce rôle de barrière étant assuré par l'épiderme, c'est celui-ci qui doit être restauré le plus rapidement possible. Le recouvrement de la lésion par les cellules épithéliales s'effectue par migration et prolifération des kératinocytes à partir des bords de la plaie. Une fois le recouvrement de la plaie terminé, les kératinocytes se différencient pour former les différentes couches de l'épiderme et permettent à celui-ci de retrouver sa fonction de barrière. Beaucoup de données sont disponibles à propos de ces mécanismes [1-7]. Cependant, le mode de migration des kératinocytes dans la plaie n'était encore récemment expliqué que par des hypothèses sans preuve formelle $[8,9]$.

Grâce au génie tissulaire, nous avons construit un modèle tridimensionnel de plaie cutanée [10] (Figure 1). Pour commencer, nous avons reconstitué une peau humaine dont les caractéristiques sont très proches de la peau in vivo [11]. Pour cela, nous avons extrait les cellules humaines provenant du derme, les fibroblastes, et celles provenant de l'épiderme, les kératinocytes. Les fibroblastes ont été cultivés en présence d'acide ascorbique pendant 28 jours. Les cellules sont alors entourées de matrice et forment un feuillet manipulable. Deux de ces feuillets ont été superposés afin de former un derme sur lequel ont été ensemencés et cultivés des kératinocytes. L'ensemble a ensuite été placé à l'interface air-liquide afin d'obtenir un épiderme bien différencié comportant toutes les couches d'un épiderme normal (Figure 2). Une plaie a alors été créée en utilisant un poinçon à biopsie de $6 \mathrm{~mm}$ de diamètre. Pour permettre aux cellules épithéliales de migrer, un $3^{\mathrm{e}}$ feuillet dermique a été ajouté sous la peau lésée avant de remettre le tout en culture (Figure 1). Les résultats obtenus ont permis de comprendre les mécanismes de la migration des kératinocytes dans la plaie. La réépithélialisation s'effectue grâce à l'intervention de deux mécanismes complémentaires, en fonction de l'emplacement des cellules dans l'épiderme en régénération.

Aux bords de la plaie initiale, les résultats histologiques ont montré une avancée de l'épiderme non lésé dans la plaie (Figure $2 \varepsilon$, entre la marge de la plaie au niveau du derme et la coupure franche de l'épiderme plus avancée dans la plaie). Cette avancée est plus importante dans les couches supérieures constituées de cellules anucléées mortes que dans les couches les plus basses contenant les cellules vivantes. Ces résultats, associés à ceux obtenus en immunohistologie, nous ont amené à conclure à une avancée de l'épiderme grâce à la pression mitotique. En effet, les cellules basales sont en perpétuelle division, engendrant un renouvellement constant de l'épiderme. Les cellules formées lors de la division, faute de place au niveau de la couche basale, montent vers les couches supérieures en se différenciant peu à peu, puis meurent avant de désquamer. L'espace libéré lors de la formation de la plaie permet aux nouvelles cellules de se déplacer non pas de bas en haut, mais de côté, produisant ainsi l'avancée épidermique visible histologiquement. Ce mécanisme n'avait jamais été noté lors des études réalisées in vivo, car la formation de croûtes sur les plaies associée à une contraction importante du derme (surtout chez les animaux) ne permettaient pas de visualiser ce phénomène.

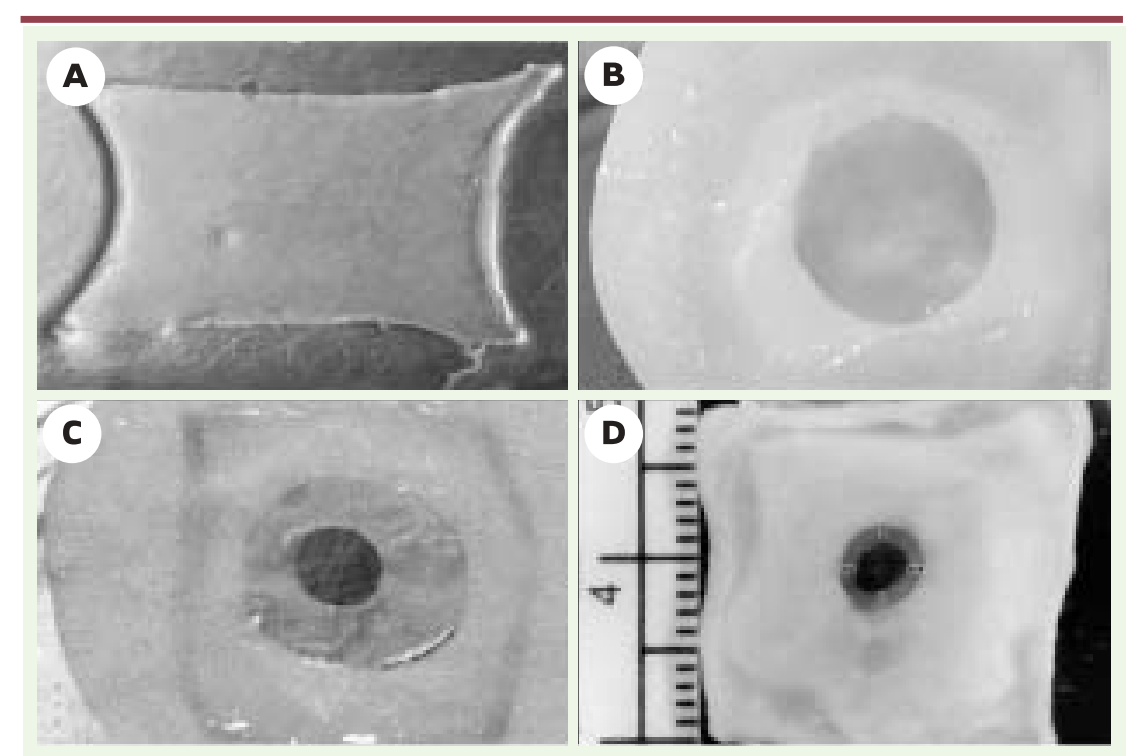

Figure 1. Fabrication du modèle de plaie par génie tissulaire (d'après [10]). A. Feuillet obtenu après 28 jours de culture. $\boldsymbol{B}$. Peau reconstruite après différenciation de l'épiderme. $\boldsymbol{C}$. Formation de la plaie et ajout d'un feuillet dermique sous la peau lésée. $D$. migration des kératinocytes sur le feuillet de migration après 3 jours de cicatrisation (flèches). 
Ce mécanisme semble peu important, mais sur une plaie de $6 \mathrm{~mm}$ de diamètre, il a permis après trois jours de recouvrir environ $30 \%$ de la surface de la plaie. II est donc probablement important à l'échelle des plaies de petite surface telles que les incisions.

Le second mécanisme conduisant à la réépithélialisation se trouve à l'extrémité de l'épiderme: il s'agit de la langue de migration. Histologiquement, la langue de migration est très fine et constituée de quelques cellules d'épaisseur. Les kératinocytes immédiatement situées derrière et au-dessus de la cellule basale la plus avancée dans la plaie sont allongés. Leurs cytoplasmes sont partiellement à hauteur de la couche suprabasale, mais la partie la plus avancée dans la plaie est en contact avec le feuillet dermique. Ces cellules suprabasales migrent donc au-dessus des cellules basales déjà en contact avec la matrice extracellulaire par un mouvement de saute-mouton (Figures 2C et 2D).

La réépithélialisation est donc un processus qui fait appel à deux mécanismes de migration cellulaire à deux endroits différents de la plaie: mécanisme de migration par feuillets sur les bords de la plaie, et migration par saute-mouton dans la langue de migration. II est fort probable que ces mécanismes soient réglés par différents facteurs de croissance [12], mais également par le type de matrice extracellulaire sur laquelle les cellules migrent. De nombreuses études restent encore à mener pour bien comprendre l'ensemble des méca-

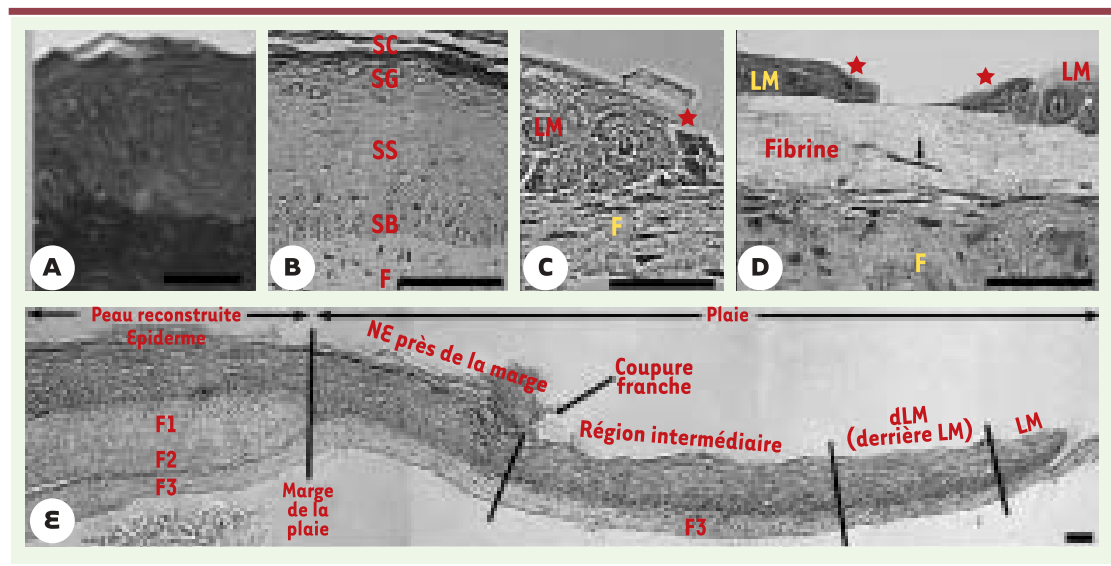

Figure 2. Histologie du modèle de cicatrisation (d'après [10]). A. Histologie d'une peau prélevée in vivo. B. Histologie d'une peau obtenue par génie tissulaire. F: feuillet dermique, SB: stratum basale, SS: stratum spinosum, SG: stratum granulosum, SC: stratum corneum; C et D. Grossissement de la langue de migration (LM) montrant les cellules suprabasales avançant sur une

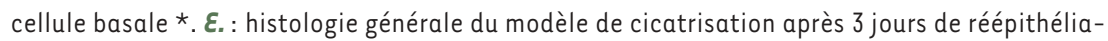
lisation. Au niveau de l'épiderme, la coupure franche réalisée lors de la formation de la plaie est décalée par rapport à celle réalisée dans le derme (marge de la plaie) montrant une avancée de l'épiderme non lésé dans la plaie. $N \varepsilon$ : néoépiderme, $\mathrm{Fl}, \mathrm{F} 2$ et $\mathrm{F} 3$ : numérotation des feuillets superposés afin de former le derme (échelle: barre $=50 \mu \mathrm{m}$ ). nismes en jeu, mais nous avons désormais, grâce au génie tissulaire, un outil permettant d'aller plus loin dans la compréhension fine de ces processus.

\section{Les cellules communiquent en permanence}

Combien de fois nous lavons-nous les mains dans une journée ? À chaque lavage, un bon nombre de cellules épithéliales de surface sont éliminées selon un processus normalement prévu par la nature. Ainsi, chaque jour, notre peau répond à cette perte cellulaire en produisant de nouveaux kératinocytes. Si ce renouvellement tissulaire semble aller de soi, il implique beaucoup d'étapes physiologiques qui dépendent, notamment, des interactions cellulaires s'effectuant entre les couches de cellules cutanées. Nous savons que les fibroblastes dermiques ont la capacité de stimuler la croissance des kératinocytes en coculture directe ou dans des peaux reconstruites [13-16]. Quels sont les facteurs mis en jeu dans ces interactions dermo-épidermiques?

Bien que la nature de tous ces facteurs ne soit pas encore connue, plusieurs cytokines sécrétées par les kératinocytes en culture ont été identifiées: TGF (transforming growth factor) $\alpha$, IL (interleukine)-1, IL-3-like-factor, IL-6, IL-8, GM-CSF (granulocytemacrophage colony stimulating factor), TNF (tumor necrosis factor) $\alpha$, IFN (interféron) $\alpha$, IGF (insulin-like growth factor) [17-19]. Des travaux ont déjà établi l'existence d'échanges de facteurs sécrétés par les fibroblastes et les kératinocytes [20, 21], tels le KGF (keratinocyte growth factor) et le FGF (fibroblast growth factor) 7. Nous avons confirmé ces observations, et sommes allés un peu plus loin par le biais du système de la coculture indirecte [22]. Cette technique a pour objectif de cultiver au moins deux types cellulaires différents, partageant le même milieu de culture mais n'étant pas en contact direct. Le principal intérêt de cette méthode est de pouvoir étudier et purifier les protéines sécrétées et échangées par les cellules afin de connaître leur nature et leurs effets.

Les travaux réalisés dans notre laboratoire ont révélé l'importance des échanges entre les cellules du derme et de l'épiderme [22]. D'abord, les kératinocytes grandissent deux à trois fois plus vite lorsqu'ils sont cultivés en coculture indirecte avec les fibroblastes. Nous savons que les kératinocytes 
requièrent normalement la présence de différents additifs dans le milieu de culture pour que leur aspect morphologique et leur taux de croissance soient maintenus in vitro [23] (Figure 3A). II a été fort intéressant de constater que les échanges de facteurs de croissance entre les différents types de cellules pouvaient remplacer les additifs habituellement essentiels au maintien de l'état des kératinocytes. En effet, en absence de sérum, d'EGF (epidermal growth factor) et d'hydrocortisone, les kératinocytes conservent leur morphologie, augmentent leur vitesse de croissance de 3 à 5 fois et forment de belles colonies compactes (Figure 3C) lorsqu'ils sont cultivés en présence de fibroblastes dermiques. En revanche, en leur absence, les kératinocytes vieillissent rapidement, comme le démontre la présence de dômes cellulaires cornifiés (blanchâtres) au centre des colonies (Figure 3B). II s'agit d'une première étape - elle est désormais franchie - vers la compréhension des interactions dermo-épidermiques, mais surtout de leur fonction sur le renouvellement de l'épiderme. Nous avons aussi mis en évidence l'induction d'un facteur de croissance de $40 \mathrm{kDa}$ sécrété par les fibroblastes dermiques, qui semble jouer un rôle dans la stimulation de la croissance des kératinocytes [22].

Ce facteur de croissance est actuellement en cours de purification, de même que d'autres facteurs produits par les cellules cutanées au sein de notre système de coculture indirecte. Nous espérons pouvoir isoler ces facteurs dans un avenir proche afin de les utiliser pour stimuler la croissance des kératinocytes et accélérer la production de feuillets épidermiques autologues pour le traitement des grands brûlés. De plus, nous pourrons optimiser la production de peaux reconstruites (épiderme et derme) en modulant les concentrations des facteurs de croissance ajoutés dans le milieu de culture, en fonction des étapes de production [13]. À la lumière de résultats publiés précédemment, il semble que les interactions épithélium-mésenchyme puissent aussi être étudiées en culture en utilisant des cellules d'origine tumorale [24-26]. II n'est donc pas utopique de penser que la découverte de nouveaux facteurs de croissance pourrait avoir de nombreuses applications thérapeutiques, non seulement en dermatologie, mais aussi dans d'autres systèmes organiques connexes.

\section{Garder un greffon vivant: un problème d'irrigation}

Une des difficultés majeures dans la transplantation d'organes reconstruits par génie tissulaire est la vascularisation des implants. En effet, lorsqu'un tissu est greffé dans l'organisme, il faut qu'il soit vascularisé très rapidement dans toute son épaisseur, sinon les portions non vascularisées se nécroseront du fait d'une nutrition, d'une oxygénation et d'une évacuation des produits du catabolisme déficientes. Or, la néovascularisation, c'est-à-dire la progression dans le greffon de nouveaux vaisseaux sanguins issus du réseau vasculaire de l'hôte, est un processus lent. Le temps nécessaire augmente avec la distance à parcourir, c'est-à-dire l'épaisseur du tissu. En transplantant sur la souris immunodéficiente des peaux reconstruites humaines d'environ $1 \mathrm{~mm}$ d'épaisseur, nous avons confirmé que la diffusion passive de nutriments pouvait palier sur de
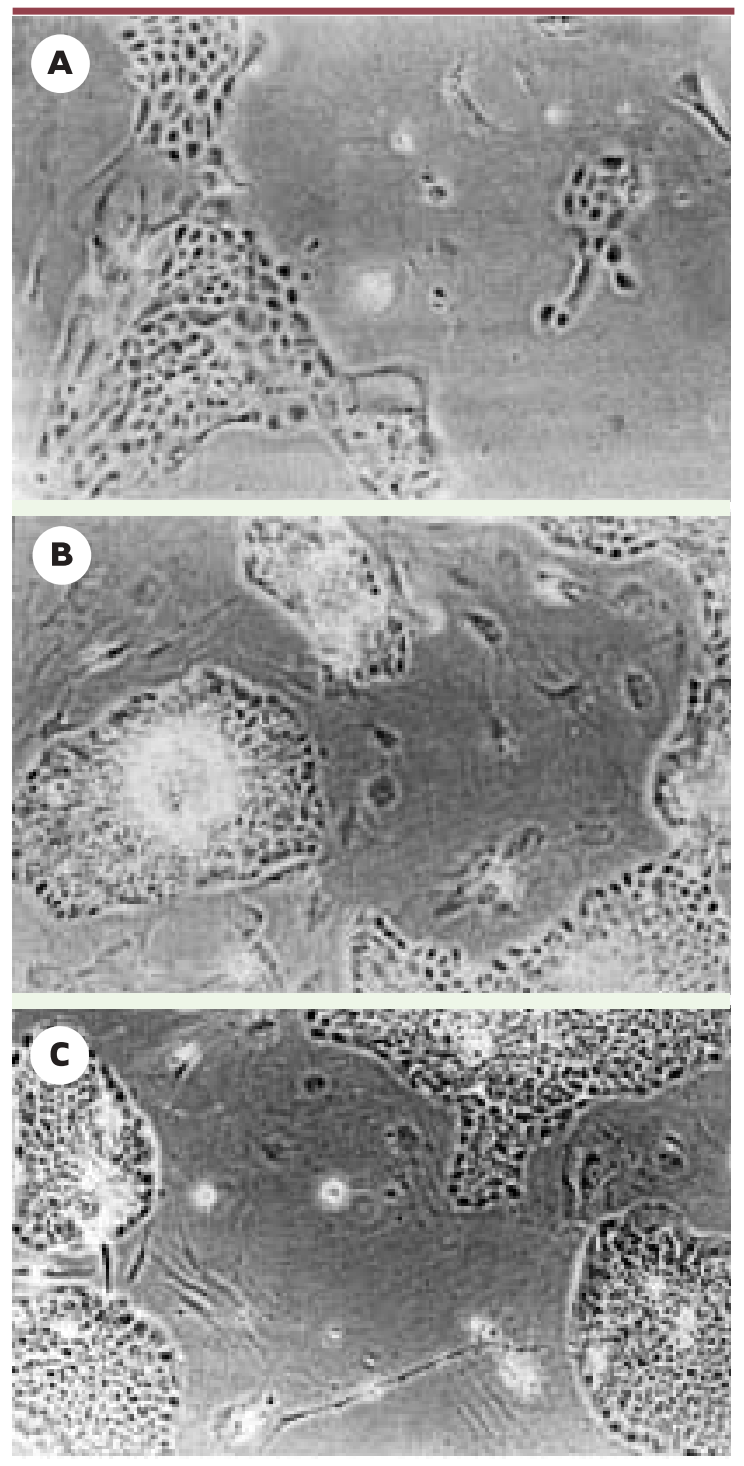

Figure 3. Rôle des fibroblastes dans la culture des kératinocytes. Culture de kératinocytes isolés de peau humaine et cultivés pendant 3 jours dans un milieu riche en suppléments ( $A$ ) ou dans un milieu pauvre, en absence $(\boldsymbol{B})$ et en présence $(\boldsymbol{C})$ de fibroblastes dermiques sur les filtres du système de co-culture indirecte. 
courtes distances l'absence d'un réseau vasculaire [27, 28]. Dans ce modèle, la reconstruction d'un réseau vasculaire dans toute l'épaisseur du greffon prend environ 15 jours, pendant lesquels la simple diffusion passive d'exsudat issu de la plaie suffit à nourrir le greffon. Cette diffusion ne permet toutefois pas de nourrir les tissus sur de grandes distances. La transplantation des peaux reconstruites, à peine plus épaisses, mais d'une surface nettement plus élevée, est accompagnée de nécrose. L'irrigation continuelle du greffon avec un milieu de nutrition en application topique est possible, mais cette solution est difficilement praticable en clinique et elle augmente notablement les risques d'infection [29]. Or, comment se fait-il qu'il soit possible de transplanter des autogreffes de peau pleine épaisseur

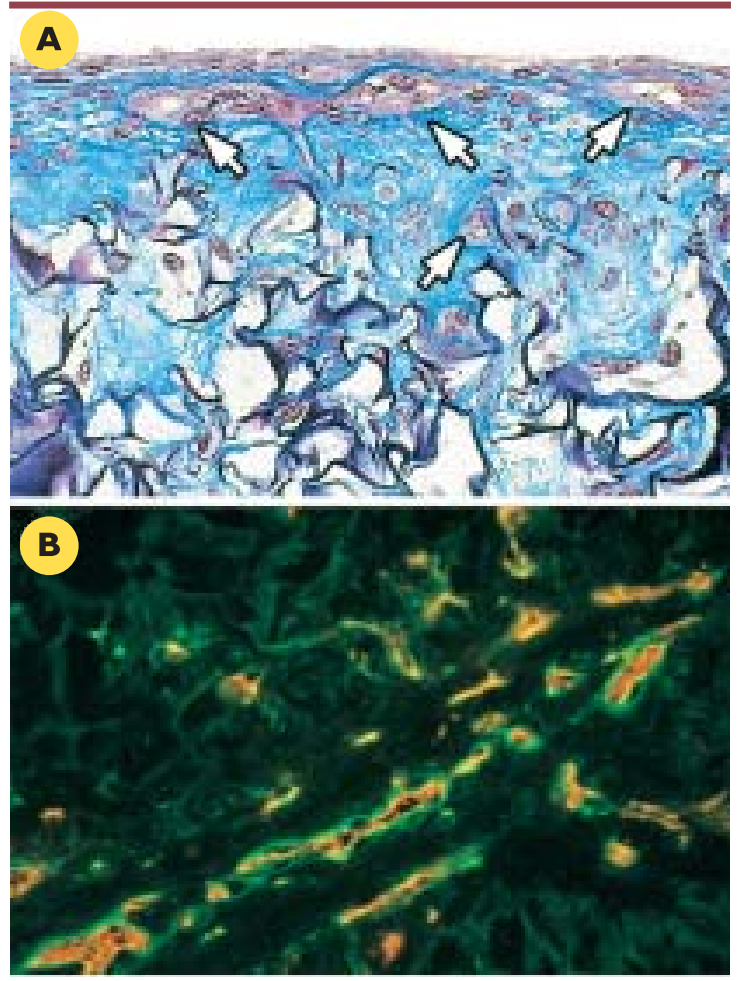

Figure 4. Formation de pseudocapillaires par les cellules endothéliales humaines dans la peau reconstruite endothélialisée. Lorsqu'elles sont cultivées avec des fibroblastes humains dans une éponge de collagène, les cellules endothéliales forment des pseudocapillaires caractérisés par une lumière interne fermée (exempte de matériel extracellulaire) ( $\boldsymbol{A}$, flèches blanches) et tapissés de cellules endothéliales. Ces cellules sont identifiables en immunohistochimie par un marquage spécifique avec un anticorps anti-PECAM (platelet/endothelial cell adhesion molecule) - 1 ( $B$, marquage rouge). Les pseudocapillaires sont entourés d'une membrane basale dont l'un des principaux constituants est la laminine (marquage vert) (grossissement $\mathrm{x} 400$ ). (jusqu'à $5 \mathrm{~mm}$, par exemple) sans rencontrer de problème de nécrose? Lorsqu'on transplante sur une plaie un tissu contenant déjà un réseau vasculaire établi, comme c'est le cas d'une autogreffe de peau, les vaisseaux sanguins du greffon ont la capacité de se reconnecter avec le réseau vasculaire de l'hôte. II s'agit d'une anastomose spontanée des deux systèmes vasculaires, qui permet en 2 à 5 jours de rétablir une circulation sanguine dans tout le greffon, quelle que soit son épaisseur $[30,31]$ : ce phénomène s'appelle l'inosculation.

Nous avons voulu tirer profit de ce processus en mettant au point, pour la première fois, une peau reconstruite in vitro contenant un réseau vasculaire préétabli. Notre démarche a consisté à ajouter des cellules endothéliales humaines dans notre modèle de peau reconstruite constitué de fibroblastes cultivés dans une éponge de collagène, modèle qui avait déjà fait ses preuves, aussi bien in vitro qu'in vivo $[27,32,33]$. Les cellules endothéliales humaines, pourtant réputées réfractaires aux manipulations in vitro, ont spontanément formé des tubules caractérisés par une lumière interne. L'observation en microscopie électronique à transmission a démontré que les tubes étaient formés par des cellules jointives contenant des corps de Weibel-Pallade, signature caractéristique des cellules endothéliales, et que ces cellules délimitaient une lumière interne contenant quelques débris cellulaires, mais exempte de matrice extracellulaire, ce qui suggérait que ces tubes étaient clos. Ces observations ont été confirmées par des marquages en immunofluorescence utilisant des anticorps spécifiques des cellules endothéliales (comme des anticorps anti-PECAM (platelet/endothelial cell adhesion molecule)-1 ou anti-facteur von Willebrand), qui ont permis d'observer la distribution de ces structures pseudocapillaires dans le modèle de peau reconstruite endothélialisée [34] (Figure 4). L'analyse par microscopie confocale des tubes a permis de démontrer que ces pseudocapillaires observés en coupes transversales ou longitudinales ne correspondaient pas à de simples tronçons isolés, mais qu'ils formaient des ramifications dans la majeure partie de l'épaisseur de la peau reconstruite endothélialisée, ce qui indiquait qu'un réseau vasculaire tridimensionnel complexe avait été reconstruit dans un organe in vitro.

Une dernière question demeure néanmoins: ce réseau permettra-t-il d'assurer une anastomose de ses pseudocapillaires avec le réseau vasculaire de l'hôte après implantation de la peau reconstruite endothélialisée in vivo ? Nous travaillons actuellement à répondre à cette interrogation. Les expériences préliminaires réalisées indiquent que la présence de ce réseau préétabli permet 
d'accélérer la vitesse de revascularisation et que la reconnexion des pseudocapillaires avec la circulation sanguine de l'hôte est possible, selon des modalités comparables à celles observées avec une greffe de peau humaine. Un tel résultat est extrêmement encourageant et aura un impact majeur dans le développement du génie tissulaire, en permettant de produire des organes plus épais sans courir le risque qu'ils subissent une nécrose du fait d'une insuffisance de vascularisation.

\section{Les cellules souches: usines du vivant}

Véritable usine de fabrication de l'épithélium cutané, les cellules souches, grâce à leur grand potentiel de multiplication, assurent le maintien de l'épiderme dont le renouvellement s'effectue tous les 28 jours. L'étude des cellules souches a pour objectif de mieux comprendre les mécanismes de renouvellement normal des tissus, ainsi que leur régénération après une blessure.

Les données expérimentales suggèrent que les cellules souches de l'épithélium cutané sont situées dans le renflement des follicules pileux, pour les endroits du corps où la peau contient des poils, et dans le creux des papilles dermiques de la peau glabre (paume des mains et plante des pieds) $[35,36]$ (Figure 5). L'identification d'une protéine structurale, la kératine 19, présente dans le cytoplasme des cellules souches, mais généralement absente dans les autres cellules de l'épithélium cutané, nous a permis de mieux les caractériser [37]. Cette kératine procure des propriétés particulières aux cellules qui l'expriment [38]. Les résultats montrent que la peau des enfants contient un plus grand nombre de cellules souches que celles des adultes. Ainsi, la quantité d'épithélium pouvant être produite à partir d'une petite biopsie de la surface d'un timbre est beaucoup plus grande pour un enfant $\left(1 \times 10^{32}\right.$ cellules, pouvant couvrir $1 \times$ $10^{17} \mathrm{~km}^{2}$ ) que pour un adulte (quelques mètres carrés seulement) [39].

Le génie tissulaire a permis de confirmer l'importance de la membrane basale, structure permettant l'attachement de l'épiderme au derme afin d'assurer la conservation des cellules souches dans la couche basale. En effet, une migration anormale de cellules souches vers la surface de l'épiderme, conduisant à leur perte, est observée dans les équivalents cutanés produits par la méthode des gels de collagène dans des conditions où la membrane basale n'est pas complètement structurée [40]. En revanche, les peaux reconstruites par la méthode d'autoassemblage présentent une membrane basale complète (Figure 5D), qui favorise l'attachement des cellules souches comme l'attachement des cellules de l'épiderme au derme.

Le développement de méthodes favorisant la conservation des cellules souches en culture a permis la production d'épidermes, cultivés à partir de cellules autologues (c'est-à-dire provenant du patient), destinés à la greffe des grands brûlés (Figure 5B) [41-43]. Ces cultures sont greffées sur les plaies afin de régénérer l'épiderme de façon permanente. La nature autologue des cellules prévient le rejet des cellules épidermiques, qui sont immunogéniques. L'utilisation de cellules souches embryonnaires produira des organes allogéniques et, par conséquent, ne résoudra pas les problèmes associés au rejet des greffes. C'est pourquoi il est primordial de poursuivre l'étude des cellules souches présentes chez les adultes. De plus, les cellules souches embryonnaires se différencient facilement en plusieurs types de cellules, et la maîtrise parfaite de

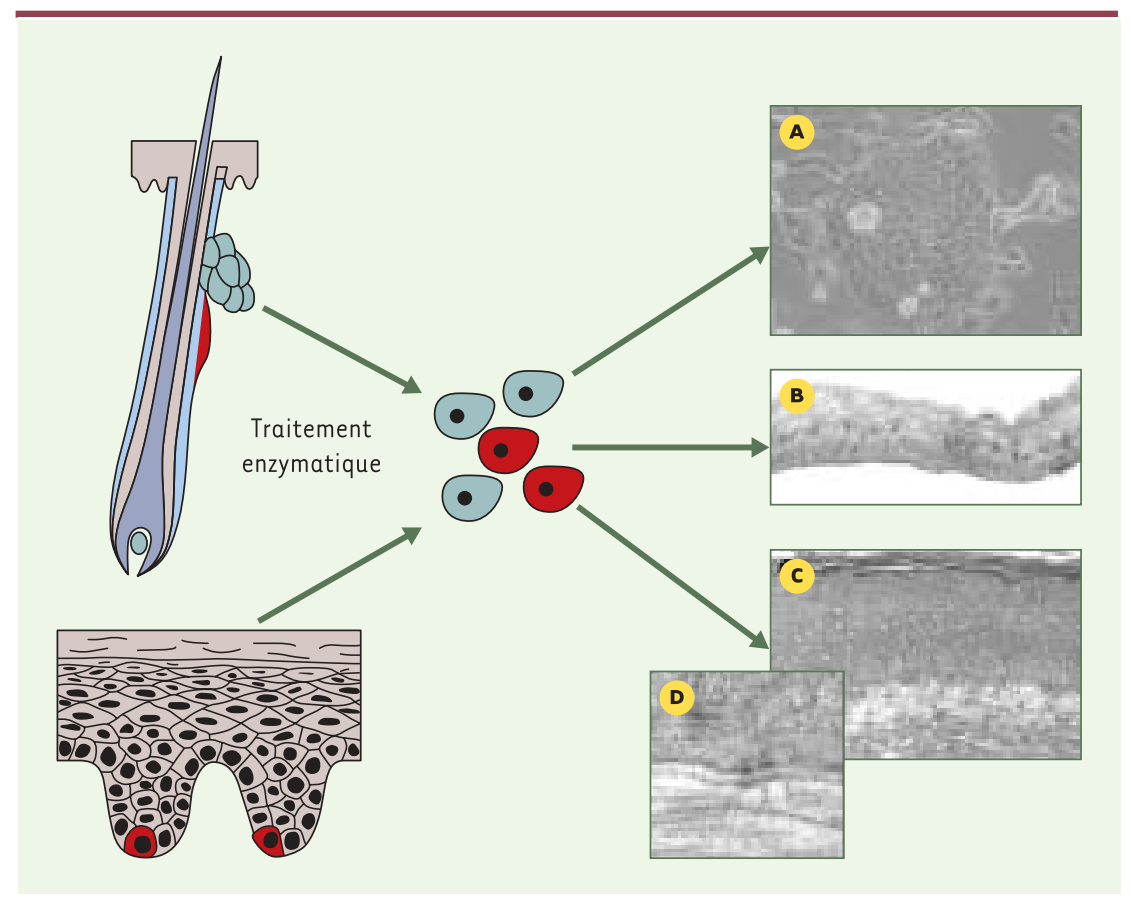

Figure 5. Cellules souches cutanées et peaux reconstruites. Les cellules souches cutanées localisées dans le follicule pileux ou la peau glabre expriment la kératine 19 (en rouge). À partir d'une simple biopsie, les cellules souches peuvent être extraites et mises en culture (A), produire des feuillets épidermiques greffables $(B)$ et être utilisées pour la genèse de structures plus complexes telles que les peaux reconstruites par génie tissulaire (C), contenant une jonction dermo-épidermique ou membrane basale bien structurée (D) (schéma de Danielle Larouche, LOEX). 
leur différenciation afin d'obtenir le type cellulaire désiré est l'un des défis qu'il faudra surmonter avant de les utiliser en clinique.

Les modèles développés par génie tissulaire fournissent de nouveaux outils pour l'étude des caractéristiques des cellules souches, ainsi que de leur potentiel de différenciation in vitro. Ils pourront s'appliquer à d'autres organes, notamment à la cornée. La persistance des cellules souches dans les tissus reconstruits et la possibilité d'y transférer des gènes lors de la culture in vitro ouvrent des perspectives d'application en thérapie génique.

\section{Conclusion}

Le génie tissulaire est un nouveau domaine ayant permis de franchir une nouvelle étape dans l'étude des mécanismes physiologiques du vivant. Cette approche permet d'étudier en profondeur des mécanismes délicats à mettre en évidence in vivo ou in vitro avec des cultures en monocouche. Cette compréhension ouvrira de nouvelles voies thérapeutiques dont devraient bénéficier un grand nombre de patients de demain. $\Delta$

\section{RÉFÉRENCES}

1. Zhao M, Song B, Pu J, Forrester JV, McCaig CD. Direct visualization of a stratified epithelium reveals that wounds heal by unified sliding of cell sheets. FASEB J 2003; 17: 397-406.

2. Falanga V, Grinnel $F$, Gilchrest B, Maddox YT, Moshell A. Experimental approaches to chronic wounds. Wound Repair Regen 1995; 3 : 132-40.

3. Garlick JA, Taichman LB. Fate of human keratinocytes during reepithelialization in an organotypic culture model. Lab Invest 1994; 70: 916-24.

4. Genever PG, Cunliffe WJ, Wood EJ. Influence of the extracellular matrix on fibroblast responsiveness to phenytoin using in vitro wound healing models. $\mathrm{Br} J$ Dermatol 1995; 133: 231-5.
5. Jansson K, Kratz G, Haegerstrand $A$. Characterization of a new in vitro model for studies of reepithelialization in human partial thickness wounds. In Vitro Cell Dev Biol Anim 1996; 32: $534-40$.

6. O'Leary R, Arrowsmith M, Wood $\varepsilon$ J. The use of an in vitro wound healing model, the tri-layered skin equivalent, to study the effects of cytokines on the repopulation of the wound defect by fibroblasts and keratinocytes. Biochem Soc Trans 1997; 25 : 369S.

7. Stephens P, Wood $\varepsilon$ J, Raxworthy MJ. Development of a multilayered in vitro model for studying events associated with wound healing. Wound Repair Regen 1996; 4 : 393-401.

8. Buck RC. Cell migration in repair of mouse corneal epithelium. Invest Ophthalmol Vis Sci 1979 . 18: 767-84.

\section{SUMMARY}

Tissue engineering : a tool to understand the physiological mechanisms

Tissue engineering is a new domain, which allows some very unique studies of many human physiological mechanisms. This technology, based on cell capacity to reproduce a three-dimensional tissue with or without the help of biomaterials, is an interesting approach to study cells in an environment quite similar to the in vivo context. This article summarizes the LOEX's (laboratory of experimental organogenesis) scientific endeavor in tissue engineering in order to better understand some physiological or pathological mechanisms. Thus wound healing, stem cells, graft vascularization and cell interactions are domains where tissue engineering has already made a significant impact. $\Delta$

9. Ortonne JP, Loning T, Schmitt D.

Immunomorphological and ultrastructural aspects of keratinocyte migration in epidermal wound healing. Virchows Arch 1981; 392: 217-30.

10. Laplante A, Germain L, Auger F, Moulin V. Mechanisms of wound reepithelialization: hints from a tissue-engineered reconstructed skin to longstanding questions. FASEB J 2001; 15: 2377-89.

11. Michel M, L'Heureux N, Pouliot R, Xu W, Auger FA, Germain L. Characterization of a new tissue-engineered human skin equivalent with hair. In Vitro Cell Dev Biol Anim 1999; 35: 318-26.

12. Werner S, Peters KG, Longaker MT, Fuller-Pace F, Banda MJ, Williams LT. Large induction of keratinocyte growth factor expression in the dermis during wound healing. Proc Natl Acad Sci USA 1992; 89: 6896-900.

13. Auger FA, Pouliot R, Tremblay N, et al. Multistep production of bioengineered skin substitutes: sequential modulation of culture conditions. In Vitro Cell Dev Biol Anim 2000; 36: 96-103.
14. Coulomb B, Lebreton C, Dubertret L. Influence of human dermal fibroblasts on epidermalization. J Invest Dermatol 1989; 92: 122-5.

15. Moulin V, Auger FA, Garrel $D$, Germain L. Role of wound healing myofibroblasts on reepithelialization of human skin. Burns 2000; 26: 3-12.

16. Bouvard V, Germain L, Rompre P, Roy B, Auger FA. Influence of dermal equivalent maturation on a skin equivalent development. Biochem Cell Biol 1992; 70: 34-42.

17 Matsue H, Cruz PD, Jr., Bergstresser PR, Takashima A. Cytokine expression by epidermal cell subpopulations. J Invest Dermatol 1992; 99 : 42S $5 \mathrm{~S}$.

18. Tavakkol A, Elder JT, Griffiths CEM, et al. Expression of growth hormone receptor, insulinlike growth factor 1 (IGF-1) and IGF-1 receptor mRNA and proteins in human skin. J Invest Dermatol 1992; 99: 343-9.

19. Phan TT, Lim IJ, Bay BH, et al. Role of IGF system of mitogens in the induction of fibroblast proliferation by keloid-derived keratinocytes in vitro. Am J Physiol Cell Physiol 2003; 284: c860-9. 
20. Kratz G, Haegerstrand A, Dalsgaard C-J. Conditioned medium from cultured human keratinocytes has growth stimulatory properties on different human cell types. J Invest Dermatol 1991; 97: 1039-43.

21. Palmieri C, Roberts-Clark D, Assadi-Sabet A, et al. Fibroblast growth factor 7 , secreted by breast fibroblasts, is an interleukin-lbeta-induced paracrine growth factor for human breast cells. J Endocrinol 2003; 177: 65-81.

22. Goulet F, Poitras $A$, Rouabhia M, Cusson D, Germain L, Auger FA. Stimulation of human keratinocyte proliferation through growth factor exchanges with dermal fibroblasts in vitro. Burns 1996; 22: 107-12.

23. Rheinwald JG, Green H. Cell serial cultivation of strains of human epidermal keratinocytes: the formation of keratinizing colonies from single cells. Cell 1975; 6: 331-43.

24. Wang CS, Goulet F, Lavoie J, et al. Establishment and characterization of a new cell line derived from a human primary breast carcinoma. Cancer Genet Cytogenet 2000; 120: $58-72$.

25. Wang CS, Goulet F, Tremblay N, Germain L, Auger FA, Têtu B. Selective culture of epithelial cells from primary breast carcinomas using irradiated $3 T 3$ cells as feeder layer. Pathol Res Pract 2001; 197: 175-81.

26. Wang CS, Goulet F, Auger F, Tremblay N, Germain L, Tetu B. Production of bioengineered cancer tissue constructs in vitro: epithelium-mesenchyme heterotypic interactions. In Vitro Cell Dev Biol Anim 2001; 37 : 434-9.
27. Berthod F, Germain L, Li H, Xu W, Damour 0, Auger FA. Collagen fibril network and elastic system remodeling in a reconstructed skin transplanted on nude mice. Matrix Biol 2001 . 20: 463-73.

28. Warburg $\varepsilon$. Versuche an überlebendem carcinomgewebe. Biochem Stschr 1923; 142: 317-33.

29. Boyce ST, Supp AP, Harriger MD, Greenhalgh DG, Warden GD. Topical nutrients promote engraftment and inhibit wound contraction of cultured skin substitutes in athymic mice. J Invest Dermatol 1995;

104: 345-9.

30. Young DM, Greulich KM, Weier HG. Species-specific in situ hybridization with fluorochrome-labeled DNA probes to study vascularization of human skin grafts on athymic mice. J Burn Care Rehabil 1996; 17: 305-10.

31. Supp DM, Wilson-Landy K, Boyce ST. Human dermal microvascular endothelial cells form vascular analogs in cultured skin substitutes after grafting to athymic mice. FASEB J 2002: 16: 797-804.

32. Berthod F, Sahuc F, Hayek D, Damour 0, Collombel C. Deposition of collagen fibril bundles by long-term culture of fibroblasts in a collagen sponge. J Biomed Mater Res 1996; 32: 87-94.

33. Berthod F, Germain L, Guignard R, et al. Differential expression of collagens XII and XIV in human skin and in reconstructued skin. J Invest Dermatol 1997; 108: 737-42.
34. Black AF, Berthod F, L'Heureux N, Germain L, Auger FA. In vitro reconstruction of a human capillary-like network in a tissue-engineered skin equivalent. FASEB J 1998; 12: 1331-40.

35. Cotsarelis G, Sun TT, Lavker RM. Label-retaining cells reside in the bulge area of pilosebaceous unit: implications for follicular stem cells, hair cycle, and skin carcinogenesis. Cell 1990; 61: 1329-37.

36. Lavker RM, Sun T-T. Heterogeneity in epidermal basal keratinocytes: morphological and functional correlations. Science 1982; 215: 1239-41.

37. Michel M, Török N, Godbout M-J, et al. Keratin 19 as a biochemical marker of skin stem cells in vivo and in vitro : keratin 19 expressing cells are differentially localized in function of anatomic sites, and their number varies with donor age and culture stage. J Cell Sci 1996; 109: 1017-28

38. Fradette J, Germain L, Seshaiah P, Coulombe PA. The type I keratin 19 possesses distinct and context-dependent assembly properties. J Biol Chem 1998; 273: 35176-84.
39. Germain L, Michel M, Fradette J, Xu W, Godbout MJ, Li H. Skin stem cell identification and culture: a potential tool for rapid epidermal sheet production and grafting. In: Rouabhia $M$, ed. Skin substitute production by tissue engineering: clinical and fundamental applications. Austin: R.G. Landes Publishers, 1997: 177-210.

40. Michel M, L'Heureux N, Auger FA, Germain L. From newborn to adult: phenotypic and functional properties of skin equivalent and human skin as a function of donor age. J Cell Physiol 1997; 171 : 179-89.

41. Damour 0, Braye F, Foyatier J, et al. Cultured autologous epidermis for massive burn wounds: 15 years of practice. In: Rouabhia M, ed. Skin substitute production by tissue engineering: clinical and fundamental applications. Austin: R.G. Landes Publishers, 1997 : 23-45.

42. Auger FA. The role of cultured autologous human epithelium in large burn wound treatment. Transpl Impl Today 1988; 5: 21-4.

43. Green H, Barrandon Y. Cultured epidermal cells and their use in the generation of epidermis. News Physiol Sci 1988; 3: 53-6.
TIRÉS À PART

V. Moulin 